\title{
Observed Behaviors of a Child while using an Adapted Ride-on Car: A Case Study
}

\section{Plummer T*}

Department of occupational therapy, Belmont University, USA

${ }^{*}$ Corresponding author: Teresa Plummer, Department of occupational therapy, Belmont University, 6807 Burkitt Rd, USA, Tel: 6159304888; Email: teresa.plummer@belmont.edu

\section{Case Report}

Volume 2 Issue 1

Received Date: January 17, 2019

Published Date: February 08, 2019

DOI: $10.23880 /$ aphot- 16000119

\section{Abstract}

There is a dearth of literature on the use of augmented mobility with infants and young children. However, there have been several studies that indicate that the use of a modified ride-on-toy enriches a child's access to the environment. A few case studies have been done with children with cerebral palsy, spinal muscular atrophy, spina bifida, or down syndrome, but no case study has been done with a child with Segawa Syndrome. This case study aims to add to the existing literature on the observed behavior of a child while using a modified ride-on toy. Data was collected through use of video recordings of 10 minutes of play on a mat, and 10 minutes of play in the modified ride-on toy, for 8 weeks. Data was collected every two weeks, coded by two researchers, and behavior frequencies were collected. During each session, the child's performance was scored on a modified power mobility skills checklist. After 8 weeks of use of the modified ride-on car, the child's score increased on the modified power mobility skills checklist, and had greater environmental interaction than before the study. Preliminary results indicate that a child with a mobility impairment can benefit from using a modified ride-on car and experience greater opportunities to engage in their environment.

Keywords: Behavior; Power Mobility; Play; Segawa Syndrome

Abbreviations: DRD: Dopa-responsive Dystonia.

\section{Introduction}

Through play, a child is able to develop motor and social skills, increase participation, and enhance quality of life $[1,2]$. Not only is play an important part of a child's development, it is a right [3]. Children with mobility impairments have an increased risk of encountering barriers to play opportunities [4]. Recent research studies have shown that the use of a power mobility device can increase a child's participation in play activities [5]. As a result of power mobility, a review of the literature suggests that a child will exhibit increased behaviors such as environmental interaction [4,6-9], social participation $[5,10]$, and emotional expression [11].

In recent years, the use of modified or adapted ride-on cars has been used to as an alternative for children with mobility impairments to eliminate potential barriers both in home and in the clinic [12-15]. Research is still limited in regard to early power mobility, specifically modified ride-on cars.

The proposed study aims to: 1) explore the influence of a modified ride-on car on the behavior of a child with a mobility impairment; and 2) refine the use of the 


\section{Annals of Physiotherapy \& Occupational Therapy}

observational checklist and determine whether the findings are reflected in the scores from the power mobility skills checklist.

\section{Methodology}

Approval for this study was granted by the Internal Review Board at Belmont University and was conducted with the help of the School of Occupational Therapy and School of Physical Therapy at Belmont University College of Health Sciences.

\section{Research Design}

Similar to previous studies $[13,15]$ this study uses a single subject case study design to follow a single individual and track the response to power mobility over time. A case study design was chosen because the intervention (i.e. the modifications to the batteryoperated power mobility device) was unique to this participant and was not compared to a control [16].

\section{Materials and Measures}

Environment: All data collection was recorded at the same facility for each of the sessions, either in a therapy gym, private therapy room, or outdoors on the playground. During free exploratory play, the child was placed on a mat in the therapy gym with a variety of sanitized, plastic toys. Any potential barriers or hazards were removed or avoided to maintain participant safety. During recording of time in the power mobility device, the participant operated the device in one of two locations: 1 ) in the therapy gym, or 2) outdoors in a fenced in playground area. The playground consisted of balls, climbers, swings, bikes, and other children.

Power mobility device: The power mobility device used for this study was a modified ride-on toy. Modifications were made to this device to provide greater postural support and changing the location of the activation switch easy access. The caregivers added a commercial child fivepoint harness that provided more stability after the initial modifications were made, which ended up being a better option for this participant.

Power mobility skills checklist: The power mobility skills checklist used in this study was a modified version found in previous studies $[17,18]$. Items that referenced navigating a curb, office space, or joystick were not used in this study and overall was modified to be appropriate for a battery-operated power mobility device, in this case a modified ride-on toy. See Appendix A.
Observational checklist: In a previous, unpublished pilot study, the researchers created a checklist of behaviors typically seen during a child's engagement with a power mobility device. This checklist was created by emailing experts in the field of power mobility, and having each expert rank the ten most common behaviors witnessed when children operate a power mobility device. Based on the responses, the researchers consolidated the behaviors into seven themes: emotional expression, sensory seeking, language/vocalization, physical response, environmental interactions, attention regulation, and social interaction. For this study, only the themes and the definitions were use for coding purposes.

\section{Procedure}

Once informed consent was received from the participant's caregiver, video recording began. The researchers used high definition video recording devices to record 20 minutes of observation, beginning with a baseline week and continuing every other week thereafter for eight weeks. The first session (baseline), consisted of fitting the power mobility device to the child, allowing the child to become familiar with the device, and begin to explore the functions of the device. For every recording session, ten minutes of play time on a mat and ten minutes of play in a ride-on toy were collected. To avoid predictability, mat and ride-on toy recordings were alternately collected first every two weeks as well.

During the recording, another researcher scored the participant on the Power Mobility Skills checklist. During the weeks that the participant was not on site for data recording, their caregiver was asked to fill out a log for each time the child and caregiver used the ride-on toy at home, how long it was used, and a brief note describing anything significant during each documented use.

\section{Results}

\section{Participant}

Participant A is a 21-month-old female diagnosed with TH deficient Dopa-responsive Dystonia (DRD), otherwise known as Segawa Syndrome. Segawa Syndrome is characterized by dystonic hypertonus, which can lead to frequent falls and fluctuating dystonia throughout the day [19]. Based on the child's health intake form, A used an infant stroller as her primary means of mobility at the time of the study, required constant support during sitting and could not hold head up for extended amounts of time (less than two minutes), and had delayed motor development. 


\section{Annals of Physiotherapy \& Occupational Therapy}

\section{Data Analysis}

Using an adapted protocol, $[20,21]$, the researchers broke each video into 15 second intervals, and only viewed the first five seconds of each interval for coding purposes. Qualitative data analysis was conducted within Nvivo [22] and coded by two researchers. Two researchers coded $100 \%$ of each data point for each set of recordings to establish interrater reliability. For this participant, researchers agreed on the observed behavior $83.78 \%$ of the time, (number of agreements/total number of intervals $=\%$ of agreement). In order for an interval to be coded, the behavior needed to be present for greater than $50 \%$ of the five second interval. In cases when two equally present behaviors were observed, both were counted as long as both researchers agreed.

During baseline recording, the most frequently recording behavior while the child was playing on the mat was attention regulation, followed by environmental interaction. The most frequent behavior recorded in the car was emotion-negative, followed by attention regulation. Week two was highlighted by a significant drop in negative emotional expression while in the car, with no reoccurrences of this behavior for the next four session. The participant engaged with her environment more during week two and the subsequent weeks, compared to baseline, both on the mat and in the car. Attention regulation while in the car remained consistent throughout each session, with a peak occurrence in the car occurring at week six with 13 . During week six, the participant had seven total occurrences of language/vocalization, four while in the car, and three while on the mat. The most occurrences prior to this week, occurred during the baseline session with only two. Another important behavior, was social interaction. This behavior did not occur while the child was in the car during baseline, but was recorded every session after with a peak occurrence of eight during week two. While on the mat, however, this participant was consistent with displaying this behavior throughout all five sessions, with a peak occurrence rate of six during week four.

The participants score on the adapted power mobility skills checklist increased after every session. During baseline, A scored a 10, followed by 21 for week two, 26 for week four, 28 for week six, and finally a 32 for week 8 .

\section{Discussion}

The primary focus of this study was twofold: 1) explore the influence of a modified ride-on car on the behavior of a child with a mobility impairment; and 2) refine the use of the observational checklist and determine whether the findings are reflected in the scores from the power mobility skills checklist.

Of the seven themes originally identified at the beginning of the study, the three behaviors that were coded the most frequently were over the five data collection sessions were environmental interaction, attention regulation, and social interaction. From those three themes, environmental interaction had the most significant change during the duration of the study. Figures 1-3 shows that Participant an increasingly interacted with her environment more as she became more comfortable with her car, which reinforces the review of the literature that states access to power mobility would enable a child with a mobility impairment to interact more with their environment [4,6-9].

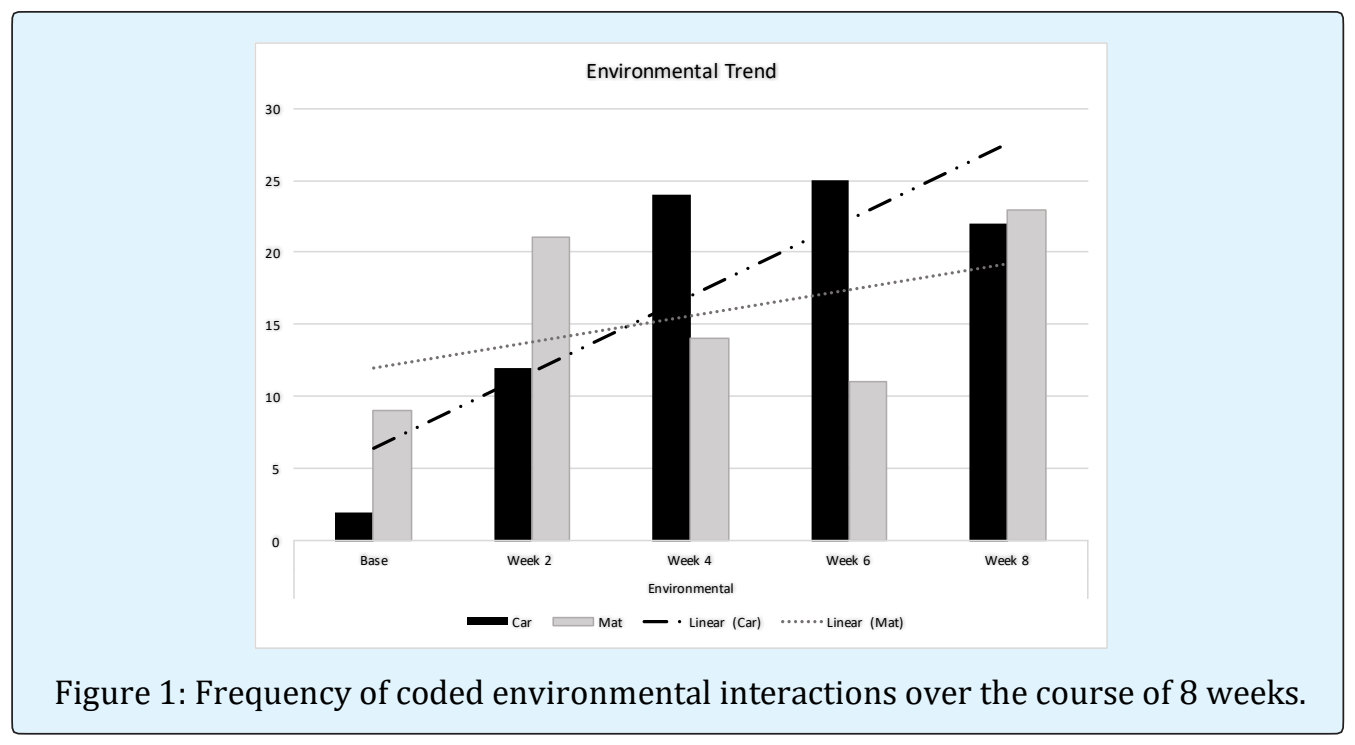

Plummer T. Observed Behaviors of a Child while using an Adapted Ride-on Car: A Case Study. Ann Physiother Occup Ther 2019, 2(1): 000119. 


\section{Annals of Physiotherapy \& Occupational Therapy}

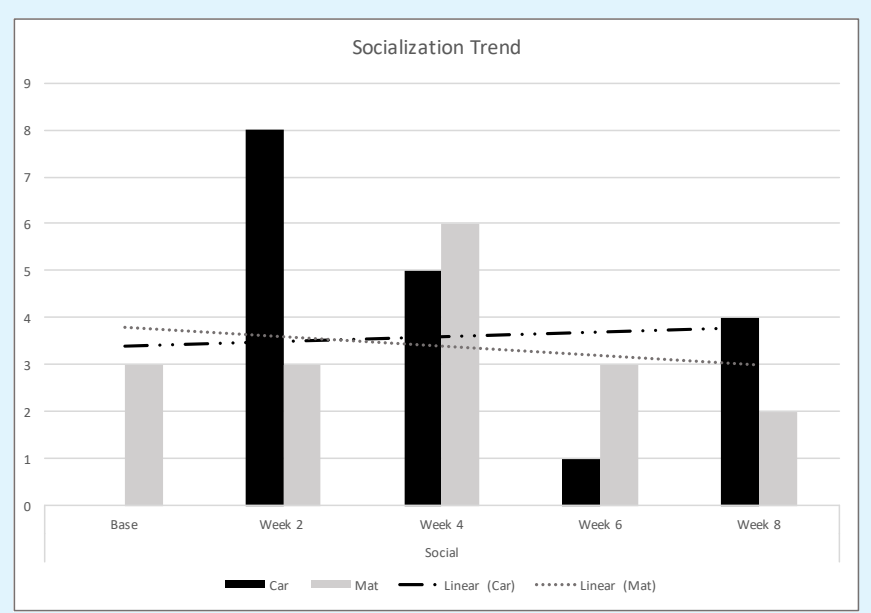

Figure 2: Frequency of coded social interactions over the course of 8 weeks.

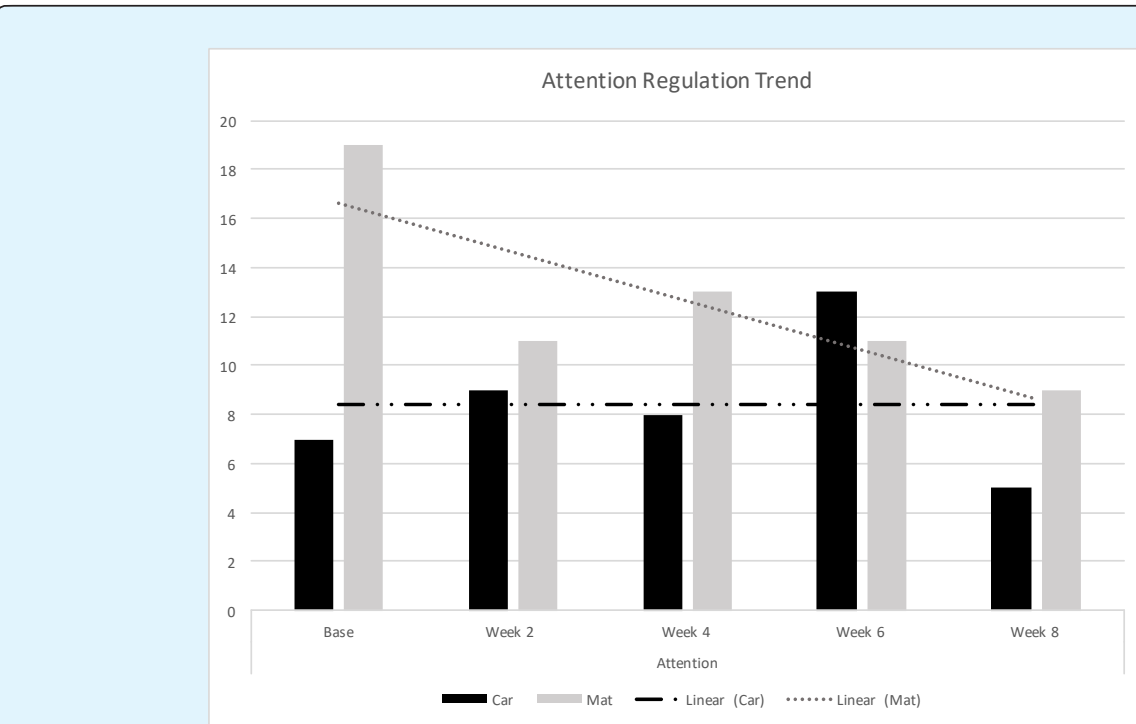

Figure 3: Frequency of coded attention regulation responses over the course of 8 weeks.

Attention regulation appears to drop as the study progresses, however the researchers hypothesize that this trend is due to the increase in the frequency of environmental interaction, rather than a true decrease in attention.

While socialization does not appear to have much change during the 5 sessions, the participant did increase significantly from car baseline to week two. This increase is likely due to the participant becoming more familiar with the car, as evidenced by the lack of negative emotional expression post-baseline. A's caregiver was present during all recording sessions and interacted more with him than the researchers. When A was in the car driving around the playground, other children would run up to her car and interact with her and she would wave and smile back.

\section{Limitations}

As with any study, there are limitations that must be addressed. One of the main limitations for this study was the design: case-study. While case-studies are important for further research, in the end the results cannot be generalized to a larger sample. Another limitation that 


\section{Annals of Physiotherapy \& Occupational Therapy}

arose during the study, was the observational checklist. The researchers improved on the interrater reliability by coding $100 \%$ of the data and coming to a consensus on any disagreements, but more research should be done to assess reliability with other researchers using the same checklist and method.

\section{Future Research}

Future research is needed to further explore the behaviors exhibited during battery operated power mobility training. Future studies should consider using larger sample sizes and a similar coding technique to strengthen the qualitative data of early power mobility. After coding data for this participant, the researchers discussed eliminating or combining themes, since some themes were never coded or were too similar to other themes.

\section{Conclusion}

Occupational and physical therapist endeavor to facilitate development of skills and functions to allow a child to access their environment, engage in play and socialize with others. For a child with mobility impairments such as Segawa Syndrome modified access to play and mobility may become necessary as the child' physical abilities deteriorate. Therefore, the use of modified ride-on-toys or augmented mobility devices may allow on-going opportunities for mobility and hence exploration, development and socialization. The goal of this study was to contribute to the growing body of knowledge regarding child use of battery operated power mobility. Children with mobility impairments should be allowed to participate in their environment, interact with their peers, and do what children do best...play! Power mobility devices are expensive, especially for children who may outgrow a device as they grow, which can lead to the child never using it again. For this reason, an inexpensive and modifiable alternative to power mobility is needed. As this study shows, adapted ride-on cars can allow a child to interact with their environment, socialize with their peers, and attend to a variety of stimuli. "A" showed steady improvement with interacting with her environment over the course of the eight weeks, as well as improvement with operated the ride-on car.

Modifying battery operated toy cars facilitates collaboration from a variety of interdisciplinary groups. First and foremost, the group centers around the child and their caregivers, as well as occupational and physical therapists, electrical engineers, mechanical engineers, computer science, robotics, and the people in the local communities. There is no set or fixed way that modifying ride-on-toys should be conducted. This innovative approach capitalizes on the skill set of all team members involved. However, the therapist may be best suited to make recommendations for modification, determine the type of access to the switch and recommendations for postural supports. Further, therapist can provide the necessary environmental support including access to playgrounds, school settings and homes to allow children to engage with peers in a variety of contexts. Additionally, recognizing the need for alternative and augmentative mobility spurs innovation, discovery and development of suitable long term solutions for children with mobility impairments. Therefore future research is needed to determine how children access mobility devices, as well as how children experience new found and/or re-gain accessibility. As the collection of studies grow regarding modified ride on toys, the need for individuals with knowledge and expertise outside the traditional occupational or physical therapist roles grows as well.

\section{Acknowledgement}

We would like to thank the parents and children who so graciously offered their time and energy into making this project a success. The authors would also like to thank the pediatric therapy clinic for allowing us to use the playground and gym for data collection.

\section{References}

1. Sonday A, Gretschel P (2015) Empowered to play: A case study describing the impact of powered mobility on the exploratory play of disabled children. Occupational Therapy International 23(1): 11-18.

2. Graham NE, Truman J, Holgate H (2015) Parents understanding of play for children with cerebral palsy. Am J Occup Ther 69(3): 1-9.

3. United Nations (1989) Convention on the rights of the child.

4. Welsh M, Bailey J (2010) Potential and possibilities: Model for providing children with disabilities access to benefits of play experiences. The National Lekotek Center.

5. Guerette P, Furumasu J, Tefft D (2013) The positive effects of early powered mobility on children's psychosocial and play skills. Assistive Technology 25(1): 39-48. 


\section{Annals of Physiotherapy \& Occupational Therapy}

6. Campbell PH, Milbourne S, Dugan LM, Wilcox MJ (2006) A review of evidence on practices for teaching young children to use assistive technology devices. Topics in Early Childhood Special Education 26(1): 313.

7. Casey J, Paleg G, Livingstone R (2013) Facilitating child participation through power mobility. British Journal of Occupational Therapy 76(3): 158-160.

8. Livingstone R (2010) A critical review of powered mobility assessment and training for children. Disability and Rehabilitation: Assistive Technology 5(6): 392-400.

9. Ragonesi CB, Galloway JC (2012) Short-term, early intensive power mobility training: Case report of an infant at risk for cerebral palsy. Pediatric Physical Therapy 24(2): 141-148.

10. Rosen L, Arva J, Furumasu J, Harris M, Lange M, et al. (2009) RESNA position on the application of power wheelchairs for pediatric users. Assist Technol 21(4): 218-225.

11. Durkin J (2009) Discovering powered mobility skills with children: 'responsive partners' in learning. International Journal of Therapy \& Rehabilitation 16(6): 331-341.

12. Galloway J, Ryu J, Agrawal SK (2008) Babies driving robots: Self-generated mobility in very young infants. Intelligent Service Robotics 1(2): 123-134.

13. Logan SW, Huang HH, Stahlin K, Galloway JC (2014) Modified ride-on car for mobility and socialization: Single-case study of an infant with down syndrome. Pediatr Phys Ther 26(4): 418-426.

14. Logan SW, Feldner HA, Galloway JC, Huang HH (2016) Modified ride-on car use by children with complex medical needs. Pediatr Phys Ther 28(1): 100-107.
15. Kenyon LK, Farris JP, Gallagher C, Hammond L, Webster LM, et al. (2017) Power mobility training for young children with multiple, severe impairments: A case series. Physical \& Occupational Therapy in Pediatrics 37(1): 19-34.

16. Taylor RR (2017) Critically appraising and classifying published and presented research. In: Kielhofner G, (Eds.), Kielhofner's Research in occupational therapy: Methods of inquiry for enhancing practice, $2^{\text {nd }}$ (Edn.), Davis FA, Philadelphia, pp: 47-58.

17. Jones MA, McEwen IR, HansenL (2003) Use of powered mobility for a young child with spinal muscular atrophy. Phys Ther 83(3): 253-262.

18. Furumasu J, Guruette P, Teft D (1996) The development of a powered wheelchair mobility program for young children. Technology and Disability 5(1): 41-48.

19. Gordon N (2007) Segaway's disease. International Journal of Clinical Practice 62(6): 943-946.

20. Brown WH, Pfeiffer KA, McIver KL, Dowda M, Almeida MJ, et al. (2006) Assessing preschool children's physical activity: The observational system for recording physical activity in children-preschool version. Res Q Exerc Sport 77(2): 167-176.

21. Logan SW, Schreiber M, Lobo M, Pritchard B, George L, et al. (2015) Real world performance: Physical activity, play, and object-related behaviors of toddlers with and without disabilities. Pediatric Physical Therapy 27(4): 433-441.

22. Nvivo qualitative data analysis software (2012) QSR International Pvt. Ltd. Melbourne, Australia. 